Rev Chil Salud Pública 2012;

Vol 16 (1): $38-46$

Tema de actualidad

\title{
Bioética crítica en salud pública ¿aguijón o encrucijada?
}

\author{
CRITICAL BIOETHICS IN PUBLIC HEALTH: THORN OR \\ CROSSROADS?
}

Introducción

La bioética, como toda disciplina joven, brega por su reconocimiento y busca su nicho cultural. El mundo académico le brinda respeto no exento de autocomplacencia por haber encontrado un nuevo campo de reflexión pero, como ética aplicada que es, aspira a la aceptación en las prácticas sociales de su competencia. La hospitalidad que le brinda la clínica, contrasta con la reticente incorporación mostrada por el quehacer científico y con sus diferencias de estilo frente a la ecoética. Su orientación hacia la salud pública es incipiente, mostrando desde muy temprano que el desarrollo teórico y la experiencia práctica alcanzada en la clínica y en la investigación biomédica, no son aplicables sin más a los complejos problemas de las diversas aristas y facetas de la salud pública. Queda claro que los aspectos normativos de la bioética constituyen una parte menor en un quehacer colectivo, donde la dualidad agente/afectado se diluye en dimensiones políticas y sociales más resistentes al análisis ético que las relaciones interpersonales. Se reconoce, por ende, la necesidad de desarrollar una amplia reflexión acerca de la vinculación de la bioética con los diversos quehaceres de la salud pública.

Por su parte, la salud pública ha sido históricamente regida por fuerzas políticas y económicas, habiéndose visto requerida y aun sobrepasada por nuevos contextos sociales y por emergencias circunstanciales -giros epidemiológicos, epidemias, enfermedades emergentes, bioterrorismo-. La epidemiología se ha rendido en gran medida al positivismo de la ciencia contemporánea, registrando los factores sociales y culturales que inciden en salud y enfermedad, pero sin cuestionar mayormente el statu quo y la tendencia al business as usual que marcan los procesos económicos que dominan en el mundo contemporáneo. Al reconocer que la agenda de la salud no se agota en la investigación empírica ni en la gestión de políticas sanitarias, pues se ve involucrada en valores fundamentales que afectan desde el bienestar individual y la estabilidad social hasta la supervivencia de poblaciones severamente desmedradas, la salud pública requiere incorporar una mirada ética amplia y permanente que reflexione y, eventualmente, ayude a rediseñar su quehacer.

\section{Voces de cuestionamiento}

La presente revisión intenta ratificar la necesidad de vincular sistemática y activamente la reflexión bioética con los complejos problemas de una salud pública que debe bregar por su ascendiente ante un proceso de desgaste del Estado y la reducción de sus tareas tradicionales.

El texto tiene la pretensión de establecer la necesidad de enfoques bioéticos en las proyecciones y realizaciones de la salud pública. Contando cada vez con menos apoyos gubernamentales estables, recurre cada vez más a la integración transnacional -salud global-, a los esfuerzos de las ONG y a las iniciativas humanitarias, como Médecins Sans Frontières, y al capital social

(1)Escuela de Salud Pública. Facultad de Medicina. Universidad de Chile. Independencia 939. Santiago. Chile. mkottow@med.uchile.cl 
como fuente de iniciativas y gestiones. Estos desplazamientos de centros que gravitan su acción, hacen necesario que hechos y valores concurran en el análisis y en las actividades, en todos los aspectos de la salud pública.

Los intrincados problemas que la salud pública contemporánea enfrenta han llevado a plantear una crisis de proporciones tales, que sugieren una revisión teórica de la disciplina y la indagación de sus fundamentos filosóficos (Nijhuis and van der Maesen, 1994). Sorprendente sugerencia en consideración a la opinión mayoritaria de que la salud pública se nutre de investigaciones que entienden la epidemiología como una "ciencia médica básica, integrada con otras ciencias médicas básicas, y progresando a lo largo de un activo proceso de fertilización cruzada con estas otras disciplinas." (Renton, 1994). La orientación positivista de las principales corrientes epidemiológicas daría origen a una fundamental debilidad en la comprensión de la dinámica social de salud y enfermedad, quedando coartada su capacidad de producir cambios en la salud poblacional (van der Maesen and Nijhuis, 2000). Aun reconociendo que la salud pública se refiere al bienestar de una colectividad y que sus problemas requieren una intervención grupal, se tiende a proponer criterios reductores que objetivan puntualmente y analizan cuantitativamente la relación costos/ beneficios y la eficacia de las medidas preventivas o terapéuticas, sin atender a los fundamentos sociales substantivos de estos problemas (Fassin, 2008).

Tanta diversidad, que además incluye la noción de "complejidad anunciada" (Tarride, 1998) y señala la futilidad de hacer distingos entre enfoques disciplinarios sociales versus científiconaturales (Carvajal, 2011), confirma la conveniencia de desarrollar una verdadera filosofía de la salud pública como fundamento sólido, y su importancia para el desempeño y las proyecciones a futuro de la salud pública (Weed, 1999). En forma simultánea, aparecen en el umbral del siglo XXI los primeros intentos de dar a luz una bioética específica para la disciplina. Los "fundamentos filosóficos de la salud pública se ordenan en aspectos ontológicos, epistemológicos y éticos." (Ibid.), que se influyen mutuamente y solo pueden ser reflexionados en conjunto: "En decisiones de salud pública, las perspectivas ontológica y ética están entramadas." (Weed 1999: 100). De allí que el giro epidemiológico hacia la "Nueva Salud Pública" y su énfasis en el individuo (Petersen and Lupton, 2000), tenga consecuencias bioéticas profundas que aún no han sido atendidas en toda su magnitud (Kottow, 2011).

\section{Fundamentos ontológicos}

Tal vez sea el uso del término "ontológico" demasiado ambicioso, por cuanto no se trata de un debate filosófico sobre la naturaleza del ser (ontos), sino de describir la diversidad de perspectivas que inspiran a la salud pública, que pueden ser de corte individualista, social (sociedad como sistema estructurado), poblacional (subsistema social), comunitario (vínculo de intereses comunes), o global.

La mirada ontológica pregunta por la realidad, o la construcción de conceptos como salud, enfermedad, público, y devela que los enfoques de la salud pública varían según la preponderancia que recibe ora el individuo y su agregación poblacional -Pareto, Weber-, ora la sociedad como una realidad orgánica que se constituye en un sistema social compuesto de subsistemas o conjuntos grupales -Marx, Durkheim- (van der Maesen and Nijhuis, 2000). Cada una de estas perspectivas tiene, a su vez, variantes que determinan el modo cómo la salud pública conoce e interviene en la realidad.

Hay un problema ontológico más de fondo. Se ha señalado que mientras el quehacer médico se concentre en el cuerpo, no habrá sino una filosofía de la biología, mas no de la medicina, hasta que la medicina acepte que su objeto de atención es que los seres humanos tienen un cuerpo vivo al mismo tiempo que son un cuerpo viviente (Spicker, 2000, p. 19). En forma análoga, la salud pública perderá fertilidad en tanto no abandone los conceptos abstractos y entienda su objeto de estudio e intervención como la Lebenswelt, el mundo de la vida, que Haberlas -basado en Husserl- describiera como una realidad colectiva en la que viven los individuos en tres dimensiones: la objetiva o relacionada con la realidad, la social o de interacción con los demás, y la psicológica o subjetiva. Con este entendimiento se evitan las distorsiones de pensar 
a los individuos como mónadas vivientes, a la sociedad como agregación de individuos, y al mundo como poblado de entes naturales, inertes o vivos equívocamente clasificados como agentes o pacientes.

\section{Principios epistemológicos: disputas conceptuales}

Los aspectos epistemológicos de la salud pública se refieren principalmente a la epidemiología, pero también se relacionan con el conocimiento que subyace a la promoción en salud y a la gestación de políticas públicas sanitarias. El acercamiento de la salud pública a las disciplinas sociales y la crítica al positivismo que inspira gran parte de la investigación epidemiológica, traen al recuerdo la llamada "disputa positivista" que hace 50 años animara el debate de la sociología alemana, desde una vehemente contraposición entre Popper, secundado por Albert, y la Escuela de Frankfurt representada por Adorno y Habermas. Esa polémica resulta de actualidad para la epidemiología por cuanto la "epistemología popperiana", como despectivamente la llama Karhausen, continúa siendo materia de controversia, defendida por unos (Koch, et al., 2006) y rechazada por otros (García, 2003).

Popper es epistemólogo, su racionalismo crítico se ejerce sobre teorías elaboradas y conclusiones adelantadas, difiriendo profundamente de la teoría que Adorno caracteriza como una crítica no al método de investigación sociológica, sino a las incongruencias y contradicciones que presenta la sociedad. No es la indagación sino el objeto indagado, el que debe ser disecado y corregido, señala la Escuela de Frankfurt. Habermas dirá que la Teoría Crítica se enfoca en la "Lebenswelt" y no en la forma o método con el cual ese mundo de la vida es observado e interpretado.

La falsación de hipótesis y el rechazo del inductivismo que Popper pone como piedra angular de su epistemología, caen en el vacío al reconocer que "las disciplinas orientadas hacia la acción, como la medicina y la epidemiología, buscan certezas de sentido común y no incertidumbres lógicas", utilizando tanto elementos inductivos como deductivos, de modo que "la filosofía de Popper tiene poco que ofrecer a epidemiólogos y otros investigadores médicos" ( $\mathrm{Ng}, 1991)$.

Visiblemente, la epidemiología en sus diversas modalidades -clínica, social, molecularconstituye una búsqueda empírica de causalidades, empleando el refinamiento epistemológico de un depurado método científico y una acuciosa validación estadística. Con ello, epidemiología y salud tienden a ignorar "las realidades sociales de la vida cotidiana moderna... La salud pública moderna considera problemas modernos de relaciones sociales y económicas, nuevas formas de desigualdades, exclusiones y marginalización de muchos grupos de habitantes urbanos" (Van der Maesen y Nijhuis, 1999), enfatizadas (Vandenbroucke, 1994), pero haciendo la salvedad que "los factores socioeconómicos son 'difícilmente modificables' y considerados 'excesivamente políticos"', al punto que "utilizamos tecnología más y más avanzada en tanto que las grandes causas sociales de enfermedad permanecen ignoradas." (Pearce, 1996).

Se perfila la necesidad de reorientar la salud pública desde una disciplina basada en estudios epidemiológicos de corte científico-natural, a una de orden sociológico. Ciertamente, la mayoría de los teóricos propone un enfoque multifacético, reticular o estratificado que haga uso tanto del rigor científico como de la sensibilidad social y ecológica (Krieger and Zierler, 1996), (Susser and Susser, 1996a). En la práctica, no obstante, las perspectivas aparecen congeladas en una continuada acumulación de datos empíricos, enmarcados en un reconocimiento ya rutinario de las determinantes socioeconómicas de salud y enfermedad, que toman un carácter natural e inamovible ilustrado por preferir el término "determinantes" sobre el de "condicionantes" con el cual se podría postular la disposición a posibles modificaciones.

\section{Problemas de método}

Aquí se debate, entre otros aspectos, la insoluble contradicción de que a más rigurosa validación interna, menor es el poder de extrapolación o validez externa (Vitora), la noción de causalidad y su relación con la inferencia lógica popperiana (Renton), la hegemonía de los estudios randomizados controlados (RCT $=$ randomi- 
zed, controlled trials), la validez del metanálisis de publicaciones, la utilidad y aplicación de desarrollar modelos contrafácticos (Kaufman and Cooper, 1999; Muntaner 1999), el valor cognitivo de encuestas, los problemas de sensibilidad y especificidad, el enfoque bayesiano, y muchos otros. Estas controversias confirman "que la epidemiología en buena parte sufre de confusión teórica... el grueso de la energía en todas las ramas de la ciencia se disipa en la documentación de observaciones triviales" (Cooper and Kaufman, 1999).

\section{Relevancia de la investigación epidemiológica}

Las controversias epistemológicas derrumban otros postulados que Popper defendiera en la disputa positivista: la creencia en la correspondencia entre verdad y realidad, y la relevancia como uno de "diversos problemas extracientíficos" (Popper 1972, cursiva en original), donde habría que distinguir "aquellos intereses que no pertenecen a la búsqueda de verdad, de los intereses puramente científicos en la [búsqueda de] verdad". Estos planteamientos son diametralmente opuestos al pragmatismo contemporáneo, que rechaza del todo la idea de conocimiento como representación de la realidad. La Teoría Crítica ya había inutilizado el concepto de verdad para las disciplinas sociales, por cuanto el investigador no es observador externo sino parte de la realidad que indaga.

La relevancia no es extracientífica, sino que motiva centralmente la investigación cuyo norte es solucionar problemas y promover intereses (sociales según Habermas, corporativos en la práctica contemporánea). El afán de Popper por decretar a la ciencia ajena e inmune a consideraciones de valor, revierte a todo lo contrario: la sociedad solo financia y sustenta investigación que es relevante para ella, vale decir, que tiene valor de sustentación de sus recursos, solución de sus problemas y corrección de sus incongruencias y asimetrías de poder (Adorno, 1972).

La salud pública reconoce las complejas dimensiones sociales y ecológicas que condicionan los estados de salud y enfermedad; mas, la epidemiología tiene dificultades en asumir la necesidad de adaptar sus métodos de estudio a los nuevos postulados. Las complejidades de los diversos niveles de realidad que interesan a la epidemiología, desde sociales, poblacionales a individuales y moleculares, así como las igualmente complejas relaciones que se postulan en etiopatogenias de enfermedades infecciosas y, más aún, en las no transmisibles, no pueden ser indagadas con planteamientos causales de tipo reduccionista (Pearce \& Merletti, 2006), requiriendo enfoques a diversos niveles (Susser and Susser, 1996b), y poniendo atención en una sociología de asociaciones que estudia redes y desmonta la dicotomía sociedad/naturaleza (Latour, 2007). EI VIH no solo infecta, además modifica una serie de relaciones sociales, de influencias de poder, de interacciones entre individuo, colectivo e instituciones, que tienen consecuencias para la inserción de una epidemia en la realidad social donde anida.

Podría hablarse de un giro ético en la investigación, que abandona el estéril canon de excelencia en busca de verdad, por el de relevancia para enfrentar y aliviar los problemas de la sociedad que designa recursos a la ciencia. Se abre un filón a la democracia participativa de la ciudadanía tanto en evaluar la pertinencia de los problemas a estudiar, como en su capacidad de aporte cognitivo en lo que viene denominándose "epidemiología popular". Ya en Weber se encuentra el reconocimiento que los problemas provienen de la sociedad, son entregados a la ciencia para su estudio, volviendo a la sociedad para aquilatar la conveniencia y oportunidad de implementar las soluciones propuestas. Este proceso gana en fluidez y pertinencia si el estamento social y el científico trabajan en conjunto, estableciendo los "foros híbridos" de legos y expertos que transitan juntos desde la percepción de problemas, a la cognición de causas, y a la elaboración de políticas públicas paliativas y terapéuticas (Callon, et al., 2001).

\section{Aspectos éticos}

"¿Dónde está la ética de la salud pública?" (Mann, 1997). Esta pregunta no se refiere a la corrección moral del quehacer de la disciplina; más bien, indaga por su sensibilidad a los valores comprometidos en salud y enfermedad, que la salud pública debe reconocer y cultivar, para 
dar sentido a su actividad. "El mundo de la salud pública no tiene un conjunto de guías éticas razonablemente explícitas... El problema central es uno de coherencia e identidad: la salud pública no puede desarrollar una ética en tanto no alcance claridad acerca de su propia identidad; la experticia técnica y metodológica no son substitutos para la coherencia conceptual." (Ibid). La observación contiene dos connotaciones negativas, ciertamente que no intencionadas. Por un lado, reconoce que la salud pública ha funcionado durante 300 años en ausencia de lineamientos éticos y, asunto más grave, reconoce que la ética sería sobrepuesta como un agregado a la coherencia e identidad de la salud, lo cual, en el lenguaje de su incipiente filosofía, significaría depurar conceptualmente la ontología y la epistemología de la salud pública para luego otorgarle legitimidad ética. La historia demuestra lo contrario: una disciplina 0 un quehacer que se desarrolla instrumentalmente sin preocuparse de su médula ética, termina por volverse indiferente e inmune al análisis moral posterior, como ocurre con la técnica, la genética, la tecnociencia nuclear.

En consecuencia, ética y bioética en salud pública deben preocuparse que ontología y epistemología de la salud pública se desarrollen en conformidad con valores morales caros a la sociedad. La incorporación de la ética, sorprendentemente tardía para una disciplina social con 300 años de presencia, se desarrolla en 4-5 lustros en tres etapas: como ética profesional, como bioética normativa $y$, prospectivamente, como bioética reflexiva.

\section{Ética profesional}

En la década de 1990 aparecen las primeras publicaciones que enfocan directamente el tema. El énfasis de una ética en salud pública se propone esencialmente delinear las responsabilidades éticas de los cultores de la disciplina y la necesidad de redactar un código deontológico de ética para epidemiólogos (Karrh, 1991), vale decir, un listado de obligaciones profesionales para la multitud de profesionales de diversas disciplinas que hacen epidemiología (Fayerweather, et al., 1991). Estas preocupaciones de ini- cio llevaron a la dictación de las Guías éticas para epidemiólogos (American College of Epidemiology Ethics Guidelines for Epidemiologists, 2000) precedidas por algunos otros documentos internacionales y por el Código de ética para la profesión de educación sanitaria (1977, 1983, 1992), que marcan la culminación de la ética profesional en investigación epidemiológica.

\section{Bioética normativa}

La estrecha perspectiva de la ética profesional comienza a abrirse a una bioética de la salud pública que apunta más allá de la preocupación por los aspectos deontológicos. Los primeros esfuerzos intentan incorporar el discurso interpersonal de la bioética clínica y los cuatro principios de Georgetown -autonomía, beneficencia, no maleficencia, justicia-, pero que difícilmente se adaptan a los problemas colectivos de la salud pública donde, muy esquemáticamente, es posible detectar dos grandes áreas de reflexión: las áreas y acciones específicas de salud pública, y el debate sobre justicia social, ecológica y global.

La bioética normativa propone un conjunto de prescripciones para regular las exigencias éticas de la investigación con seres humanos, preocupándose de los requerimientos de consentimiento y confidencialidad. Asimismo, destaca y promueve los aspectos éticos de las diversas tareas e intervenciones propias de salud pública como programas de vacunas, tamizajes y diagnóstico precoz, promoción, medicina laboral, catastros y encuestas en temas sensibles -genética, etnias, grupos marginados- asignación de recursos, elaboración de políticas públicas sanitarias, manejo de emergencias.

Las normas éticas establecidas para el quehacer de la salud pública se han institucionalizado en forma de reglamentos $y$, al ser parte de la formación profesional, se han plasmado en un currículum con directrices centrales que permanecen abiertas a la deliberación de matices y variables contextuales, al punto que la "American Public Health Association publica textos que contienen el capítulo "Instrucción ética en epidemiología y salud pública", con una contundente bibliografía (Coughlin, 2009). 


\section{Bioética reflexiva}

La agenda de la bioética normativa deja sin atender aspectos más fundamentales de la ontología y la epistemología de salud pública y de epidemiología, que necesitan ser esclarecidos por cuanto "la formulación de perspectivas filosóficas es esencial para la resolución de temas complejos en salud pública." (Leed, 1999). La urgencia de este cometido nace de la desazón sentida y la crítica expresada de que "muchos epidemiólogos han perdido el contacto con la salud pública" (Terris citado por (Weed 1995), y la acusación según la cual "la salud pública se encuentra en crisis" (Nijuis \& van der Maesen, 1994, cursivas en original).

La paradoja preventivista de Rose -una medida preventiva que trae mucho beneficio a la población ofrece poco a cada individuo participante- posiblemente señale la mayor tensión ética que la salud pública alberga en su seno, consistente en la necesidad de proponer e imponer normas sanitarias efectivas cuyo cumplimiento disciplinado atenta contra la autonomía de individuos disidentes cuando esgrimen argumentos razonables para eximirse de obligaciones públicas.

La oposición individuo/sociedad distingue la epidemiología positivista, empirista, centrada en factores de riesgo y operando desde la realidad observada -bottom up-, de una epidemiología derivada de concepciones amplias, socio-ecológicas, multidisciplinarias, social-críticas -top down-. Estas estrategias no son exclusivas ni irreconciliables (Krieger, 1999), mas tampoco pueden ser arbitrariamente intercambiadas, porque operan en diversos niveles de observación e interpretación.

La salud pública se encuentra en permanente disyuntiva entre cuidar el bien común a nivel colectivo, convocar a la participación de todos los ciudadanos cuando el buen éxito de sus acciones lo requiera -la vacunación universalmente obligatoria que logró erradicar la viruela- y, por otra parte, reconocer la autonomía de los individuos para tomar decisiones singulares y restarse a políticas impositivas -el excepcionalismo epidemiológico frente a la epidemia VIH- (Burr, 1977). La oposición entre autonomía individual y disciplina colectiva se agudiza desde el impulso con que la "Nueva Salud Pública", también conocida como Medicina Preventiva, se ha centrado en el individuo, desplazando las tradicionales tareas de la salud pública a nivel poblacional o colectivo -Medicina Social- (Kottow, 2011). Es el giro desde la salud pública poblacional a la Nueva Salud Pública que individualiza e internaliza los factores de riesgo, enfatizando la auto-responsabilidad en materias de promoción de salud y prevención de enfermedad (Graham 2010; Petersen y Lupton, 2000).

Todo apunta, no obstante, a que la salud pública, incluyendo la epidemiología en tanto su brazo científico, es en gran medida una disciplina social que debe desarrollar teorías sobre condicionantes económico-sociales de salud y enfermedad, el concepto de "calidad social" (Navarro en van de Maesen, 1999), el significado de "salud poblacional" y "enfermedad social", la denotación y connotaciones de los llamados "factores sociales de riesgo", la vigencia de la sociología de la acción, el paso de la sociología a la antropología, anunciada por Popper, y detectada en las políticas sanitarias francesas (Herzlich and Pierret, 1985; Fassin, 2004).

La bioética reflexiva trasciende los límites de su agenda disciplinar, requiriendo elementos de otros sistemas cognitivos y seduciendo a la salud pública a incluir en su horizonte consideraciones ajenas a su quehacer cotidiano. Renace la antigua aspiración de maridar el pensamiento científico con las humanidades (Nussbaum, 2000), sugerencia que por lo general no pasa de ser un basso continuo a la melodía instrumental predominante. También en la literatura especializada se encuentran llamados a la convivencia de la razón instrumental con "el núcleo de la humanidad misma" (Weed, 1995), una invocación que, al menos, hace sentido por recuperar la historia como elemento enriquecedor para entender los avatares de la salud pública contemporánea.

\section{La mirada diacrónica}

Los procesos históricos son aquellos que más sustento dan a un pensamiento pragmático. Examinar el pasado ilustra la diversidad de políticas y acciones que la salud pública ha emprendido, con lo cual queda en claro que las 
perspectivas y decisiones actuales son susceptibles de corrección si se analizan a la luz diversa de procesos anteriores. llustrativo es, por ejemplo, estudiar el período de 40 años que medió entre los estudios de Lind demostrando el valor preventivo de los cítricos para prevenir el escorbuto, hasta que el Almirantazgo Británico incorporó la fruta fresca a la dieta obligada de sus marinos. Comparado con este largo período de latencia, llama la atención que el descubrimiento de la insulina llevó dentro de un año a la elaboración y disponibilidad del medicamento para tratar la diabetes mellitus. Hay aquí enseñanzas para evaluar la lentitud de desarrollo de ciertas terapias y vacunas, comparado con el fast track de aprobación obtenido para medicamentos con altas expectativas de lucro, aunque insuficientemente ensayados.

La mirada prospectiva a lo largo del eje diacrónico devela cómo la epidemiología de riesgo no se hace cargo de su preocupación primaria consistente en "identificar y reparar problemas de salud resultantes de exposiciones pasadas, sin identificar y actuar sobre riesgos a la salud futura." (Graham 2010, p. 151). Igualmente problemática es la evaluación de acciones de salud pública mediante "estudios metodológicos" enfocados sobre resultados próximos en tiempo y espacio, dejando desatendidos las influencias sociales y ambientales que en la actualidad determinan la salud de futuras generaciones ((lbid., 157). En una época de profundas transformaciones, la epidemiología de riesgo, centrada en exposiciones específicas y conductas individuales, es "prisionera de lo proximal" y, por ende, traiciona el futuro al no considerarlo (McMichael, 1999). La sustentabilidad económica de programas de salud pública tiende a "descontar costos y beneficios futuros"; es decir, reduce sus cálculos de costos/beneficios al corto plazo inmediato. Ignorar el futuro es producto del "nuevo paradigma" que "legitima intervenciones dirigidas al individuo más que a poblaciones, a estilos de vida más que al medio ambiente, en un enfoque que ha dominado las políticas de la salud pública durante gran parte del siglo pasado" (Nathanson, 2007, citado por Graham, 2010). Sin embargo, la investigación contemporánea se encuentra frente a factores de incertidumbre cuyas proyecciones futuras permanecen ignotas, y que difícilmente se controlan mediante diseños experimentales y métodos estadísticos, toda vez que la estadística misma no solo es una herramienta analítica, sino puede a su vez ser objeto de indagación (Senra, 2008).

\section{Conclusión}

Desde que Dilthey presentara su distinción entre ciencias de la naturaleza -Naturwissenschaften- y ciencias del espíritu -Geisteswissenschaften-, se hace notar un hiato entre ambos dominios, con una clara hegemonía de la razón instrumental sobre la razón comunicativa que sufre atrición a medida que es colonizada, en el decir de Habermas, por la respetuosa pleitesía a datos y hechos a costa de desatender el aspecto de los valores. La analogía de la histórica disputa positivista enfrenta la epistemología empírica con la Teoría Crítica, con claro predominio de un cultivo positivista, cuantitativo y reduccionista de las ciencias, que ignoran las humanidades y las relegan a ser la quinta rueda del coche.

El empirismo que campea en las ciencias incluyendo la epidemiología, ha de ser ampliado y enriquecido por enfoques transdisciplinarios, de nivel múltiple, donde investigación y gestión se desenvuelven en un ambiente participativo de expertos y legos (Lascoumes, 1997). Ante todo, en las ciencias y disciplinas de la salud será fructífero dejar atrás la pleitesía a la acción basada en evidencia, incorporando un nuevo paradigma denominado ciencia post-normal que propende a un enfoque integral y crítico de incertidumbre, riesgos y valores (Funtowicz and Ravetz, 2008).

La bioética reflexiva se instala en la salud pública como una Teoría Crítica que se entiende complementaria con el positivismo reduccionista. El momento crucial del análisis crítico es el reconocimiento de la realidad social jaspeada de pliegues patológicos, condensada en el manido término de las determinantes sociales que han de ser incorporadas a la reflexión más allá de su mera mención. La actualidad es producto de la evolución histórica de una racionalidad defectuosa que rinde pleitesía a intereses que no son universales y, por lo tanto, desatienden las condiciones para la realización y la emanci- 
pación de los individuos. Llevado al lenguaje de la salud pública, significa el reconocimiento del desarrollo patológico y patogénico del entorno social y económico que causa y perpetúa el enfermar individual 0 , dicho de otro modo, debilita las funciones sociales destinadas a proteger la salud de los individuos previniendo enfermedad y promoviendo salud.

El reconocimiento de las raíces históricas se opone a la pretensión de naturalizar el entorno socioeconómico y ocultar sus efectos negativos, aletargando a la ciudadanía con altisonancias sobre felicidad, bienestar, progreso, a fin de impedir que tomen conciencia de su vida hipotecada a intereses económicos que no son los suyos. La visión crítica consiste precisamente en develar las injusticias, despertando de su letargo a los resignados inmersos en el mundo de la vida entre el poder del sistema económico y el sistema político, para que se dispongan a hacer valer sus intereses coartados, lo que ocurre "al menos por dos vías: una dirigida a aumentar el conocimiento del mundo objetivo, la otra hacia una más justa resolución de conflictos interactivos." (Honneth p. 348). En la visión pragmática de Habermas, el proceso crítico y la comunicación racional se constituyen en una defensa de intereses concretos en camino hacia la emancipación, es decir, la independencia.

Aplicada a la salud práctica, la bioética reflexiva se propone detectar las condiciones socioeconómicas dominantes y sus pliegues de injusticias, desarrollando una comunicación concertada para defender intereses ciudadanos concretos en relación a su salud frente a los abusos de la industria farmacéutica, el precario reconocimiento de un derecho y cobertura universales de necesidades sanitarias, las distorsiones de la investigación biomédica comandada por intereses corporativos e indiferente a los requerimientos locales, la medicalización y mercantilización de la medicina, la emergencia de la medicina desiderativa, el turismo médico, la hotelería hospitalaria, la interferencia de la globalización en debilitar la protección de la medicina laboral, y la reducción de las protecciones sociales a cargo del Estado.

\section{Referencias}

1. Adorno, Th.W. Zur Logik der Sozialwissenschaften. In Der Positivismusstreit in der deutschen Soziologie. T.W.e.a. Adorno, ed. Pp. 125-143. Darmastad, Neuwied: Luchterhand. 1972.

2. Burr, C. The AIDS exception: Privacy vs. public health. The Atlantic Monthly 1997 (June): 57-67.

3. Callon, M., P. Lascoumes, and Y. Barthe Agir dans un monde incertain. Paris: Éditions du Seuil. 2001.

4. Carvajal, Y. Incertidumbre en la medición de defunciones: una aproximación. Escuela de Salud Pública. Universidad de Chile. 2011.

5. Cooper, R.S., and J.S. Kaufman. Is there an absence of theory in social epidemiology? American Journal of Epidemiology 1999; 150:127-128.

6. Coughlin, S.S. Ethics in epidemiology \& public health practice. Washington, D.C.: American Public Health Association. 2009.

7. Fassin, D. Entre las políticas de lo viviente y las políticas de la vida. Hacia una antropología de la salud. Revista Colombiana de Antropología 2004; 40:283-318.

8. Fassin, D. Faire de la santé publique. C. Gamboa R., Y. (manuscrito), transl. Paris: Éditions de l'École des Hautes Études en Santé Publique. 2008.

9. Fayerweather, W.E., J. Higginson, and T.L. Beauchamp Ethics in epidemiology. Industrial Epidemiology Forum's Conference, 1991. Vol. 44 Suppl. 1, pp. 1S-169S. Journal of Clinical Epidemiology. 1991.

10. Funtowicz, S., and J. Ravetz. Post-normal science. 2008.

11. García, F.M. Popper, el contraste de hipótesis y el método crítico. Rev Cubana Salud Pública 2003; 29:5260.

12. Graham, H. Where is the future in public health? Milbank Q 2010; 88(2):149-68.

13. Herzlich, C., and J. Pierret. The social construction of the patient: patients and illnesses in other ages. Soc Sci Med 1985; 20(2):145-51.

14. Karrh, B.W. Opening remarks. J Clin Epidemiol 1991; 44(Suppl. 1):1S-4S.

15. Kaufman, J.S., and R.S. Cooper. Seeking causal explanations in social epidemiology. American Journal of Epidemiology 1999; 150:113-120.

16. Koch, E., et al. Popperian epidemiology and the logic of bi-conditional modus tollens arguments for refutational analysis of randomised controlled trials. Med Hypotheses 2006; 67(4):980-8.

17. Kottow, M., ed. Bioética y Nueva Salud Pública. Santiago. Escuela de Salud Pública. Universidad de Chile. 2011.

18. Krieger, N. Sticky. Webs, hungry spiders, buzzing flies, and fractal metaphors: on the misleading juxtaposition of "risk factor" versus "social" epidemiology. J Epidemiol Community Health 1999; 53(11):678-80. 
19. Krieger, N., and S. Zierler. What explains the public's health?- A call for epidemiologic theory. Epidemiology 1996; 7(1):107-9.

20. Lascoumes, P. La précaution un nuveau standard de jugement. Esprit 1997; 11:129-140.

21. Latour, B. Nunca fuimos modernos. Buenos Aires: Siglo Veintuno Editores. 2007.

22. Mann, J. M. Medicine and public health, ethics and human rights. Hastings Cent Rep 1997; 27(3):6-13.

23. McMichael, A. J. Prisoners of the proximate: loosening the constraints on epidemiology in an age of change. Am J Epidemiol 1999; 149(10):887-97.

24. Muntaner, C. Invited commentary: social mechanisms, race, and social epidemiology. Am J Epidemiol 1999; 150(2):121-6; discussion 127-8.

25. Ng, S. K. Does epidemiology need a new philosophy? A case study of logical inquiry in the acquired immunodeficiency syndrome epidemic. Am J Epidemiol 1991; 133(11):1073-7.

26. Nijhuis, H. G., and L. J. van der Maesen. The philosophical foundations of public health: an invitation to debate. J Epidemiol Community Health 1994; 48(1):13.

27. Nussbaum, M.C.Cultivating humanity. Cambride, London: Harvard University Press. 2000.

28. Pearce, N. Traditional epidemiology, modern epidemiology, and public health. Am J Public Health 1996; 86(5):678-83.
29. Petersen, A., and D. Lupton. The new public health. London: Sage Publication Ltd. 2000.

30. Popper, K.R. Die Logik der Sozialwissenschaften. In Der Positivismusstreit in der deutschen Soziologie. T.W.e.a. Adorno, ed. Pp. 103-123. Darmastadt, Neuwied: Luchterhand. 1972.

31. Renton, A. 1994 Epidemiology and causation: a realist view. J Epidemiol Community Health 48(1):79-85.

32. Senra, N. Pesquisa histórica das estadísticas: temas e fontes. História, Ciencia, Saúde-Manguinhos 2008; 15:411-425.

33. Susser, M., and E. Susser. Choosing a future for epidemiology: I. Eras and paradigms. Am J Public Health 1996a; 86(5):668-73.

34. Susser, M., and E. Susser Choosing a future for epidemiology: II. From black box to Chinese boxes and ecoepidemiology. Am J Public Health 1996b; 86(5):674-7.

35. Tarride, M. Saúde Pública: uma complexidade anunciada. Rio de Janeiro: Editora Fiocruz. 1998.

36. Van der Maesen, L. J., and H. G. Nijhuis. Continuing the debate on the philosophy of modern public health: social quality as a point of reference. J Epidemiol Community Health 2000; 54(2):134-42.

37. Vandenbroucke, J. P. New public health and old rhetoric. BMJ 1994; 308(6935):994-5.

38. Weed, D. L. Epidemiology, the humanities, and public health. Am J Public Health 1995; 85(7):914-8.

39. Weed, D. L. Towards a philosophy of public health. J Epidemiol Community Health 1999; 53(2):99-104. 